ONLINE APPENDIX FOR

\title{
The Causal Effect of Competition on Prices and Quality: Evidence from a Field Experiment*
}

\author{
By Matias Busso and SEbastian Galiani
}

\section{Appendix I: Price Variable Construction}

\section{A. Data Sources}

Price data were obtained from the responses to three questions (sources). First, the retail survey questionnaire included a question (Question Q1) about 15 products. Retailers were asked about the brand and price of the cheapest brand that is normally available at their stores. This question pre-specified the unit of measurement. Second, in Question Q2, retailers were asked to identify the three products that they sell the most of to program beneficiaries and to provide information about the price, brand, variety and unit of measurement for three different versions of these three products. Finally, in Question Q3, consumers were asked about their weekly expenditure and the physical amount that they bought of each of the 15 products in the last 7 days.

\section{B. Coding Varieties and Brands}

In order to code all possible combinations of brand-variety (barcodes) for each product, we pooled all three sources of information. A unique code was assigned to each combination of brand-variety for each of the 15 products. Q1 and Q3 were intended to only deal with brands. In some instances, however, survey respondents mixed brands with varieties. For some products, information about the variety could be recovered from the question even when the respondent did not identify the variety, since in some cases the brand is associated with a particular variety. This imputation of missing information was based on data obtained from the webpages for each product. Two issues warrant discussion. First, the variety of the products is often not associated with a single characteristic. This is more frequently the case for some products than for others. For instance, the variety of eggs

\footnotetext{
* Busso: Research Department, Inter-American Development Bank; 1300 New York Ave. NW, Washington, DC, 20577 (email: mbusso@iadb.org). Galiani: Department of Economics, University of Maryland; 3114 Tydings Hall, College Park, MD, 20852 (email: galiani@econ.umd.edu).
} 
could differ because of their size, yolk quality, etc. So in those cases, varieties were grouped together even though the relevant attributes differ. Second, neither retailers nor consumers provided information about varieties of bread. The previous table showed the complete list of brands and varieties for each product in our sample.

\section{Measures}

Average Price (retailers). For each retailer $i$ at time $t(\mathrm{t}=$ baseline, endline), we computed the average over all 15 products $(k)$ :

$$
P_{i t}=\sum_{k=1}^{15} W_{k} * p_{i t k}
$$

In the case of the weighted average price, $\mathrm{W}_{\mathrm{k}}$ is the share of expenditure on product $k$ (see below). In the case of the unweighted average price, $\mathrm{W}_{\mathrm{k}}=1 / 15$ for all $k$.

Average Price (consumer). For each consumer $i$ at time $t$ ( $\mathrm{t}=\mathrm{b}$ aseline, endline), we computed the average (relative) price over all 15 products $(k)$ :

$$
P_{i t}=\sum_{k=1}^{K} W_{k} *\left[\frac{p_{i t k}}{\overline{P_{k t}}}\right]
$$

In the case of the weighted average price, $\mathrm{W}_{\mathrm{k}}$ is the share of expenditure on product $k$ (see below). In the case of the unweighted average price, $\mathrm{W}_{\mathrm{k}}=1 / \mathrm{K}$ for all $k$. Many consumers did not report spending for all 15 products. To avoid differences in average prices due to bundle composition, we standardized the price of each product using its average price in the sample.

\section{Weights}

The weights $W_{k}$ for the 15 products were created using the household survey. The weights represent the share of monthly expenditure on product $k$ made by all the surveyed households at baseline. In all measures, the weights add up to 1.

The weights $W_{k}$ were compared with the results of a nationally representative survey of program beneficiaries, the Evaluation Survey of Social Protection (EEPS), which was conducted in 2010/2011. In this survey, households were queried about their expenditure on a broader set of products. Appendix Table A1 indicates the results of this comparison. The first column shows the product and the second column, the sample size. The third column shows the percentage of households that reported having consumed a given product in the previous week. The fourth column shows the average share of expenditure on each product. Panel A gives the corresponding information for the 15 products that were covered in our survey. Panel B summarizes the information about other non-perishable products that may 
be sold by small-scale retailers. Panel $\mathrm{C}$ shows the measures for other fresh or perishable products typically not sold by the retailers in question.

Several facts are worth mentioning here. First, the 15 products included in our survey account for $60 \%$ of total food expenditure. Second, the other products that are sold by the retailers under analysis represent $12 \%$ of total food expenditure. Third, most households bought these 15 products. Fourth, the weights calculated in our sample are very close to those observed in the EEPS.

\section{E. Price Validation}

In order to assess the validity of our price measures, we compare price measures obtained using retailer data with those obtained using beneficiary data (an independent source of information). For each product and brand in all the districts, we calculated an average price based on the prices reported by the retailers and by the beneficiaries.

Appendix Figure 1: Distribution of $\left(\bar{P}_{k s}^{R} / \bar{P}_{k s}^{C}\right)$

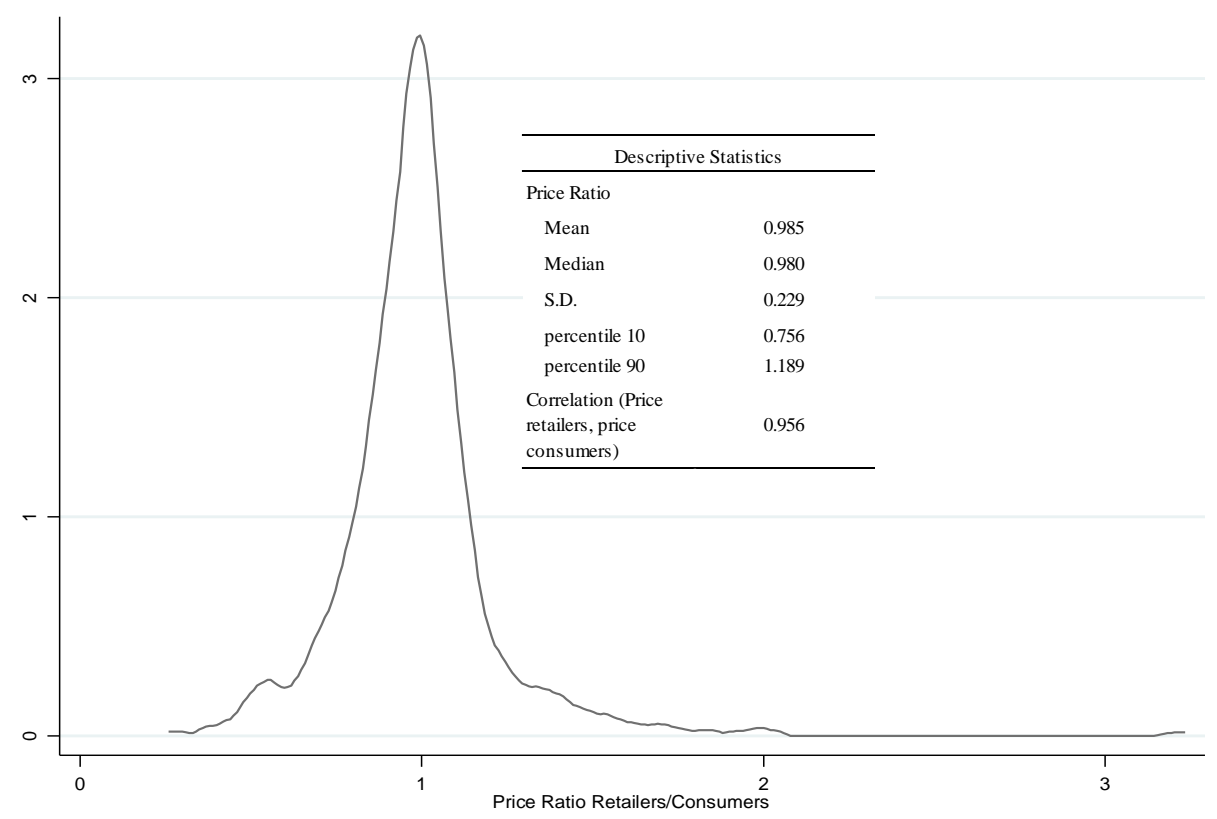

Note: The figure plots the ratio between the (simple) average price of product $\mathrm{j}$ in district $\mathrm{s}$ as reported by retailers and the (simple) average price of product $\mathrm{j}$ in district $\mathrm{s}$ as reported by consumers. The table reports statsitics that describe the distribution of that ratio and, in the last row, the correlation between those two prices. 
Let $\bar{P}_{k s}^{R}$ be the average price in district $s$ of product $k$ computed using retailer information R, which corresponds to the cheapest available option for each product. Similarly, let $\bar{P}_{k s}^{C}$ be the average price in district $s$ computed using consumers' information $\mathrm{C}$ which corresponds to the products actually bought by consumers. The average district relative price $\left(\bar{P}_{k s}^{R} / \bar{P}_{k s}^{C}\right)$ is a useful statistic for assessing how close these two measures are. Note that, without measurement error in the measures of prices, this statistic is bounded from above at 1 . The next figure shows a kernel density estimation of that price ratio. We find that the average relative price over all products and districts is 0.99 .

\section{Appendix II: Tables}

\begin{tabular}{|c|c|c|}
\hline & $\begin{array}{c}\text { At } \\
\text { baseline }\end{array}$ & $\begin{array}{c}\text { At } \\
\text { endline }\end{array}$ \\
\hline Universe of retailers in area under study & 432 & 425 \\
\hline Universe of entrant retailers & 61 & 61 \\
\hline Sample size: Retailers (in surveys) & 401 & 400 \\
\hline \multicolumn{3}{|l|}{ By type } \\
\hline Incumbent & 350 & 341 \\
\hline Entrant & 51 & 59 \\
\hline Located in targeted neighborhood & 257 & 254 \\
\hline Incumbent in targeted neighborhood & 215 & 212 \\
\hline Sample size: Beneficiaries (in surveys) & 2250 & 2118 \\
\hline \multicolumn{3}{|l|}{ By type } \\
\hline Shop in incumbent retailers & 1620 & 1563 \\
\hline Located in targeted neighborhood & 2250 & 2118 \\
\hline Number of districts & 72 & 72 \\
\hline
\end{tabular}


APPENDIX TABLE A2. EEPS 2010 - SHARE OF EXPENDINTURE ON ALL PRODUCTS

\begin{tabular}{|c|c|c|c|c|c|}
\hline \multirow[b]{2}{*}{ Product } & \multicolumn{4}{|c|}{ EEPS 2010} & \multirow[b]{2}{*}{$\begin{array}{c}\text { Survey } \\
\text { weightings }\end{array}$} \\
\hline & $\mathrm{N}$ & $\begin{array}{l}\text { Percentage } \\
\text { consumption }\end{array}$ & $\begin{array}{c}\text { Share of } \\
\text { expenditure }\end{array}$ & $\begin{array}{c}\text { Share of } \\
\text { expenditure in } \\
\text { price index }\end{array}$ & \\
\hline Fifteen survey products & & & 0.601 & 1.000 & 1.000 \\
\hline Rice & 6783 & 0.962 & 0.157 & 0.262 & 0.293 \\
\hline Chicken & 6784 & 0.784 & 0.089 & 0.148 & 0.170 \\
\hline Oil & 6786 & 0.936 & 0.059 & 0.099 & 0.094 \\
\hline Milk & 6786 & 0.338 & 0.045 & 0.075 & 0.062 \\
\hline Sugar & 6785 & 0.955 & 0.045 & 0.075 & 0.052 \\
\hline Beans & 6785 & 0.849 & 0.043 & 0.072 & 0.063 \\
\hline Salami & 6786 & 0.758 & 0.039 & 0.064 & 0.048 \\
\hline Eggs & 6785 & 0.792 & 0.030 & 0.051 & 0.050 \\
\hline Bread & 6785 & 0.755 & 0.028 & 0.046 & 0.074 \\
\hline Pasta & 6786 & 0.771 & 0.019 & 0.032 & 0.017 \\
\hline Onion & 6785 & 0.886 & 0.018 & 0.030 & 0.020 \\
\hline Cod & 6785 & 0.192 & 0.011 & 0.018 & 0.018 \\
\hline Sardines & 6786 & 0.216 & 0.009 & 0.014 & 0.014 \\
\hline Chocolate & 6784 & 0.366 & 0.007 & 0.011 & 0.015 \\
\hline Flour & 6786 & 0.278 & 0.002 & 0.003 & 0.010 \\
\hline Other non-perishable products & & & 0.121 & & \\
\hline Powdered chicken bouillon & 6786 & 0.874 & 0.025 & - & - \\
\hline Coffee & 6785 & 0.708 & 0.023 & - & - \\
\hline Water & 6786 & 0.485 & 0.017 & - & - \\
\hline Tomato paste & 6786 & 0.715 & 0.017 & - & - \\
\hline Soda & 6786 & 0.296 & 0.012 & - & - \\
\hline Smoked cutlets & 6785 & 0.142 & 0.008 & - & - \\
\hline Powdered juice & 6786 & 0.287 & 0.007 & - & - \\
\hline Ice & 6786 & 0.329 & 0.005 & - & - \\
\hline Pigeon peas & 6785 & 0.123 & 0.004 & - & - \\
\hline Dried coconut & 6785 & 0.085 & 0.002 & - & - \\
\hline Canned green beans & 6785 & 0.026 & 0.001 & - & - \\
\hline Fresh or persihable products & & & 0.264 & & \\
\hline White cheese & 6785 & 0.336 & 0.017 & - & - \\
\hline Milk & 6784 & 0.237 & 0.007 & - & - \\
\hline Yellow cheese & 6786 & 0.113 & 0.005 & - & - \\
\hline Butter & 6786 & 0.255 & 0.004 & - & - \\
\hline Orange juice & 6786 & 0.072 & 0.003 & - & - \\
\hline Plantains & 6785 & 0.723 & 0.037 & - & - \\
\hline Avocados & 6784 & 0.787 & 0.022 & - & - \\
\hline Garlic & 6785 & 0.900 & 0.022 & - & - \\
\hline Beef & 6785 & 0.240 & 0.020 & - & - \\
\hline Pork & 6785 & 0.232 & 0.019 & - & - \\
\hline Yucca & 6784 & 0.526 & 0.014 & - & - \\
\hline Green bananas & 6785 & 0.650 & 0.014 & - & - \\
\hline Chili peppers & 6782 & 0.749 & 0.009 & - & - \\
\hline Fresh fish & 6782 & 0.096 & 0.008 & - & - \\
\hline Potatoes & 6785 & 0.252 & 0.007 & - & - \\
\hline Other vegetables & 6784 & 0.604 & 0.006 & - & - \\
\hline Eggplants & 6785 & 0.303 & 0.005 & - & - \\
\hline Squash & 6785 & 0.399 & 0.005 & - & - \\
\hline Peas & 6786 & 0.134 & 0.005 & - & - \\
\hline Clupea (fish) & 6785 & 0.147 & 0.005 & - & - \\
\hline Lemons & 6783 & 0.401 & 0.004 & - & - \\
\hline Tomatoes & 6785 & 0.243 & 0.004 & - & - \\
\hline Chayote & 6785 & 0.237 & 0.003 & - & - \\
\hline Cabbage & 6784 & 0.194 & 0.003 & - & - \\
\hline Bananas & 6786 & 0.271 & 0.003 & - & - \\
\hline Carrots & 6786 & 0.175 & 0.003 & - & - \\
\hline Sweet potatoes & 6785 & 0.114 & 0.002 & - & - \\
\hline Yautia & 6785 & 0.073 & 0.002 & - & - \\
\hline Other fruits & 6786 & 0.095 & 0.002 & - & - \\
\hline Beetroot & 6785 & 0.064 & 0.001 & - & - \\
\hline Oranges & 6786 & 0.115 & 0.001 & - & - \\
\hline Mangos & 6786 & 0.055 & 0.001 & - & - \\
\hline
\end{tabular}

Note: The products in each of the three product groups are listed in descending order of share of expenditure. 
APPENDIX TABLE A3. VARIABLES

\begin{tabular}{|c|c|c|}
\hline Variable & Description & Source \\
\hline \multicolumn{3}{|l|}{ District Characteristics } \\
\hline Log (total beneficiaries - 2010) & Number of beneficiaries in January 2010 at the district level & Administrative \\
\hline Change in log (total beneficiaries -2009/2010) & Change in the number of beneficiaries at the district level from January 2009 to January 2010 & Administrative \\
\hline Log (sales -2010) & Total sales from January to May 2010 at the district level & Administrative \\
\hline Change in log (sales $-2009 / 2010$ ) & Change in total sales from January-May 2009 to January-May 2010 at the district level & Administrative \\
\hline Number of incumbent retailers 2010 & Number of active retailers per district as of February 2011 & Administrative \\
\hline Number of brands & $\begin{array}{l}\text { Average number of brands available in each distict } \\
\qquad \text { Brands }_{s, t}=\sum_{k=1}^{K} N_{k, S, t} / 15\end{array}$ & Retailer survey \\
\hline Change in $\log$ (number of retailers 2009/2010) & \# retailers 2010 - \# retailers 2009 & \\
\hline & $\overline{0.5 *(\# \text { retailers } 2010+\# \text { retailers } 2009)}$ & Administrative \\
\hline \% Solidaridad program beneficiaries / population & Solidaridad program beneficiaries as a percentage of the total population (above 18 years) & Administrative \\
\hline Average household monthly income (US\$) & Average household income in the district (above 18 years) & Household survey \\
\hline$\%$ of population with completed primary education & Percentage of beneficiaries with incomplete primary education or lower (above 18 years) & Household survey \\
\hline$\%$ of population with incomplete secondary & Percentage of beneficiaries with incomplete secondary education & Household survey \\
\hline \% Population with secondary complete or higher & Percen of beneficiariies with secondary complete or higher education & Household survey \\
\hline Urban & 1 (if district is urban) & Administrative \\
\hline District includes non-targeted neighborhoods & 1 (if district includes a non-targeted neighborhood) & Administrative \\
\hline
\end{tabular}

\section{Retailer Characteristics}

Average Price (weighted)

$\log \left(P_{i 0}\right)$, where:

$$
P_{i t}=\sum_{k=1}^{K} W_{k} * p_{i t k}
$$

$p_{i k}$ Price of product $\mathrm{k}$ in retailer $\mathrm{i}$

Household and

$W_{k}$.

$W_{k}$ Weight computed from the household survey

$W_{k}=\frac{w_{k}}{\sum_{k=1}^{K} w_{k}}$

Average Price (unweighted)

$\mathrm{K}$ is the number of products available at the store

$$
\begin{aligned}
& P_{i t}=\sum_{k=1}^{K} W_{k} * p_{i t k} \\
& W_{k}=\frac{1}{K}
\end{aligned}
$$

$\mathrm{K}$ is the number of products available at the store

Log (sales)

$$
\text { Log (self-reported sales) }
$$

Log (self-reported total number of employees)

Log (employees)
Share of CCT beneficiary customers

Number of customers - best day

Store cleanliness

Percentage of customers who, according to the retailer, are program beneficiaries

Number of customers on the best day for sales

Retailer's gender

Retailer's ownership

Retailer's education

Share of retailers in targeted neighborhood
Hygienic conditions in the store - scale of 1 to 10

Gender of retailer's owner

1 ( owns the retail store)

1 ( if retailer has more than a completed primary education)

1 (If retailer is in a targeted neighborhood) retailer surveys

Retailer survey

Retailer survey

Retailer survey

Retailer survey

Retailer survey

Retailer survey

Retailer survey

Retailer survey

Retailer survey

Retailer survey 


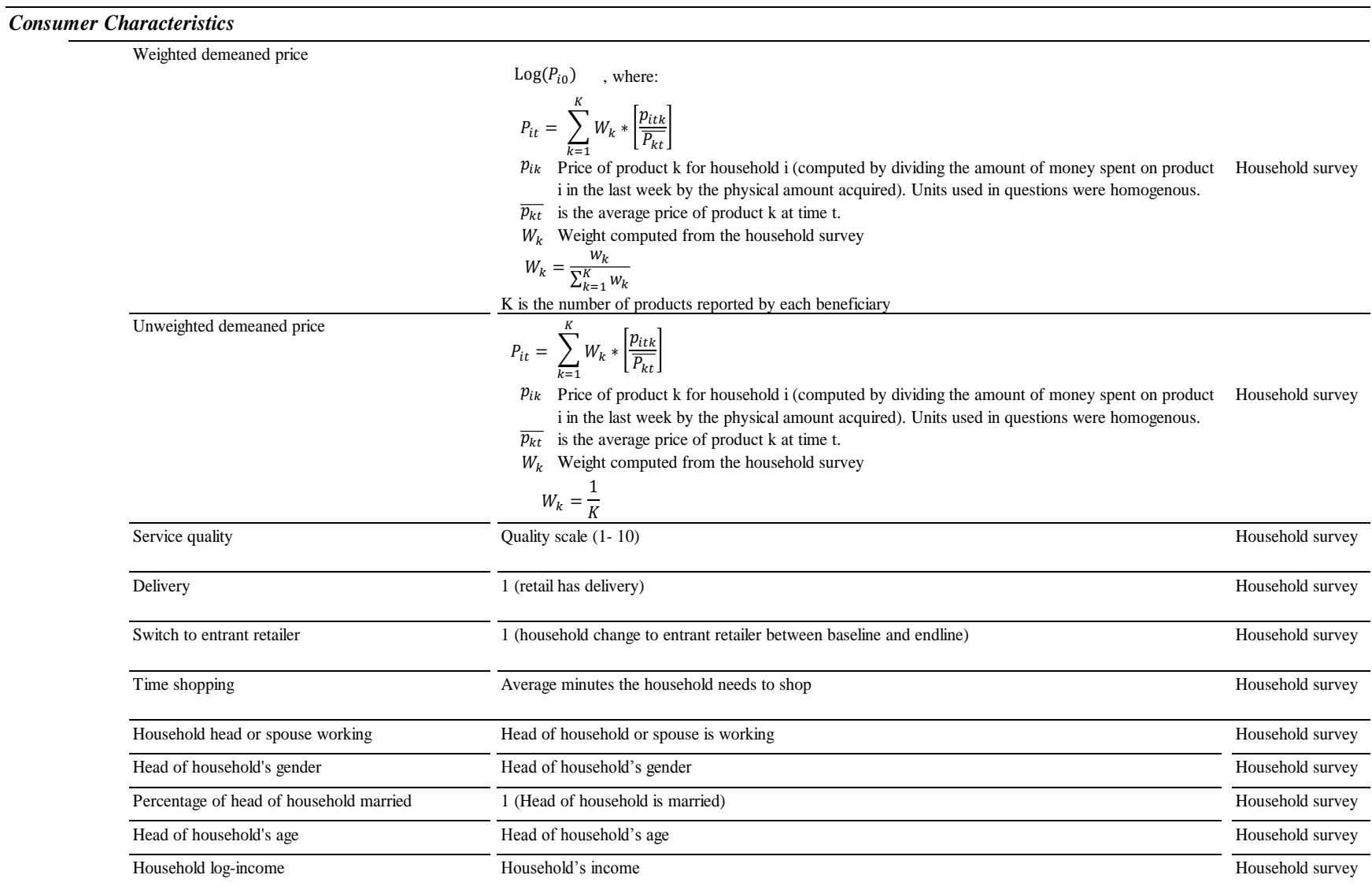

APPENDIX TABLE A4. A VERAGE CHARACTERISTICS OF ENTRANT VS INCUMBENT RETAILERS AT BASELINE

\begin{tabular}{|c|c|c|c|c|}
\hline & Entrants & Incumbents & $\begin{array}{l}\mathrm{p} \text {-value of } \\
\text { difference }\end{array}$ & $\begin{array}{c}\text { Number of } \\
\text { observations }\end{array}$ \\
\hline & [1] & [2] & [3] & [4] \\
\hline Log-price index - pre-treatment (weighted) & $\begin{array}{c}-0.343 \\
{[0.094]}\end{array}$ & $\begin{array}{c}-0.332 \\
{[0.080]}\end{array}$ & 0.443 & 400 \\
\hline Log-price index - pre-treatment (unweighted) & $\begin{array}{c}-0.258 \\
{[0.077]}\end{array}$ & $\begin{array}{c}-0.248 \\
{[0.082]}\end{array}$ & 0.388 & 400 \\
\hline 1 (retailer does special sales/promotions) & $\begin{array}{c}0.431 \\
{[0.500]}\end{array}$ & $\begin{array}{c}0.386 \\
{[0.487]}\end{array}$ & 0.527 & 401 \\
\hline Log (sales) & $\begin{array}{c}8.989 \\
{[0.904]}\end{array}$ & $\begin{array}{c}9.117 \\
{[0.821]}\end{array}$ & 0.371 & 388 \\
\hline Log (total employees) & $\begin{array}{c}1.399 \\
{[0.378]}\end{array}$ & $\begin{array}{c}1.509 \\
{[0.484]}\end{array}$ & 0.028 & 401 \\
\hline Percent male & $\begin{array}{c}0.804 \\
{[0.401]}\end{array}$ & $\begin{array}{c}0.849 \\
{[0.359]}\end{array}$ & 0.494 & 401 \\
\hline 1 (if the surveyed person is the retailer's owner) & $\begin{array}{c}0.627 \\
{[0.488]}\end{array}$ & $\begin{array}{c}0.643 \\
{[0.480]}\end{array}$ & 0.822 & 401 \\
\hline 1 (if has more than complete primary education) & $\begin{array}{c}0.686 \\
{[0.469]}\end{array}$ & $\begin{array}{c}0.623 \\
{[0.485]}\end{array}$ & 0.318 & 401 \\
\hline$\%$ Solidaridad Clients & $\begin{array}{c}49.25 \\
{[26.570]}\end{array}$ & $\begin{array}{c}48.037 \\
{[23.842]}\end{array}$ & 0.8424 & 347 \\
\hline
\end{tabular}

Note: Columns [1] and [2] report the mean and standard deviation (in square brackets) of each variable for the entrant retailers and incumbent retailers at baseline. Column [3] reports the p-value of a t-test of the difference between the two samples (using clustered standard errors at the district level). Column [4] shows the number of observations used. 


\section{Outcome}

Prices

Price index (weighted)

Price index (weighted) - Non-barcode change

Price index (unweighted)

Price index (weighted) - Random entry $=1$

Price index (weighted) - Random entry $=2$ or 3

Price index - weighted (Consumers)

Price index (weighted) of non-CCT retailers in experimental districts

Price index (weighted) of CCT retailers in non-experimental districts

Product Availability*

Percent of products that changed barcode

Percent of products that changed to a cheaper barcode

Percent of products that changed brand

Percent of products that changed variety

Service quality, Clients and Spillovers

Store cleanliness

Time shopping (minutes)

Delivery

Service-quality rating

Number of customers on best day

Share of customers CCT beneficiaries

Switch to entrant retailer*

\begin{tabular}{|c|c|c|c|c|c|}
\hline \multicolumn{2}{|c|}{ All districts } & \multicolumn{2}{|c|}{ Targeted neighborhoods } & \multicolumn{2}{|c|}{$\begin{array}{l}\text { Incumbent retailers in } \\
\text { targeted neighborhoods }\end{array}$} \\
\hline Mean & $\begin{array}{l}\text { Standard } \\
\text { deviation }\end{array}$ & Mean & $\begin{array}{l}\text { Standard } \\
\text { deviation }\end{array}$ & Mean & $\begin{array}{l}\text { Standard } \\
\text { deviation }\end{array}$ \\
\hline 0.719 & 0.058 & 0.719 & 0.059 & 0.720 & 0.058 \\
\hline 0.603 & 0.224 & 0.593 & 0.234 & 0.597 & 0.235 \\
\hline 0.782 & 0.063 & 0.783 & 0.062 & 0.784 & 0.062 \\
\hline 0.719 & 0.058 & 0.719 & 0.058 & 0.719 & 0.058 \\
\hline 0.719 & 0.058 & 0.719 & 0.058 & 0.719 & 0.058 \\
\hline- & - & 0.028 & 0.002 & 0.028 & 0.003 \\
\hline- & - & 0.727 & 0.060 & - & - \\
\hline- & - & 0.719 & 0.056 & - & - \\
\hline 0.481 & 0.124 & 0.484 & 0.126 & 0.484 & 0.126 \\
\hline 0.228 & 0.100 & 0.228 & 0.102 & 0.228 & 0.102 \\
\hline 0.446 & 0.156 & 0.452 & 0.169 & 0.452 & 0.169 \\
\hline 0.018 & 0.064 & 0.012 & 0.050 & 0.012 & 0.050 \\
\hline 7.474 & 2.023 & 7.417 & 1.992 & 7.362 & 2.011 \\
\hline- & - & 61.0 & 47.6 & 62.7 & 48.9 \\
\hline- & - & 0.454 & 0.498 & 0.464 & 0.499 \\
\hline- & - & 8.982 & 1.467 & 8.987 & 1.449 \\
\hline 106.0 & 117.9 & 113.2 & 124.9 & 113.8 & 123.8 \\
\hline 48.1 & 24.0 & 47.9 & 24.2 & 47.6 & 24.2 \\
\hline- & - & 0.041 & 0.199 & - & - \\
\hline
\end{tabular}

Note: For most variables summary statistics are measured at baseline and correspond to the samples located in treated and control districts. In the case of variables related to product availability (marked with *), which can only be defined at endline, we report the summary statistics for the control group. 


\section{APPENDIX TABLE A6. DIFFERENCES IN NON-COMPLIERS AND COMPLIERS}

\begin{tabular}{|c|c|c|c|c|}
\hline & $\begin{array}{c}\text { Compliers } \\
\text { [1] }\end{array}$ & $\begin{array}{c}\text { Non-compliers } \\
{[2]}\end{array}$ & $\begin{array}{c}\mathrm{p} \text {-value of } \\
\text { difference } \\
{[3]}\end{array}$ & $\begin{array}{c}\text { Number of } \\
\text { obs. } \\
{[4]}\end{array}$ \\
\hline \multicolumn{5}{|l|}{ A. District characteristics } \\
\hline Log (total beneficiaries - 2010) & $\begin{array}{c}6.417 \\
{[0.977]}\end{array}$ & $\begin{array}{c}6.556 \\
{[0.626]}\end{array}$ & 0.584 & 72 \\
\hline Change in log (total beneficiaries - 2009/2010) & $\begin{array}{c}0.168 \\
{[0.146]}\end{array}$ & $\begin{array}{c}0.232 \\
{[0.236]}\end{array}$ & 0.184 & 72 \\
\hline Log (sales - 2010) & $\begin{array}{l}11.285 \\
{[1.229]}\end{array}$ & $\begin{array}{l}11.295 \\
{[1.337]}\end{array}$ & 0.978 & 69 \\
\hline Change in log (sales $-2009 / 2010$ ) & $\begin{array}{c}0.971 \\
{[2.577]}\end{array}$ & $\begin{array}{c}1.774 \\
{[3.703]}\end{array}$ & 0.327 & 67 \\
\hline Number of incumbent retailers - 2010 & $\begin{array}{c}5.945 \\
{[6.753]}\end{array}$ & $\begin{array}{c}6.294 \\
{[6.469]}\end{array}$ & 0.852 & 72 \\
\hline Change in log (number of retailers - 2009/2010) & $\begin{array}{c}0.402 \\
{[0.596]}\end{array}$ & $\begin{array}{c}0.579 \\
{[0.744]}\end{array}$ & 0.316 & 72 \\
\hline$\%$ Solidaridad program beneficiaries / population & $\begin{array}{c}0.393 \\
{[0.234]}\end{array}$ & $\begin{array}{c}0.290 \\
{[0.183]}\end{array}$ & 0.099 & 72 \\
\hline Average monthly household income (US\$) & $\begin{array}{l}491.088 \\
{[86.411]}\end{array}$ & $\begin{array}{l}495.965 \\
\text { [93.582] }\end{array}$ & 0.842 & 72 \\
\hline$\%$ of population with completed primary education or lo & $\begin{array}{c}0.618 \\
{[0.080]}\end{array}$ & $\begin{array}{c}0.615 \\
{[0.067]}\end{array}$ & 0.887 & 72 \\
\hline$\%$ of population with incomplete secondary education & $\begin{array}{c}0.208 \\
{[0.052]}\end{array}$ & $\begin{array}{c}0.215 \\
{[0.048]}\end{array}$ & 0.615 & 72 \\
\hline$\%$ of population with completed secondary education or & $\begin{array}{c}0.174 \\
{[0.065]}\end{array}$ & $\begin{array}{c}0.170 \\
{[0.045]}\end{array}$ & 0.809 & 72 \\
\hline 1 (if district is urban) & $\begin{array}{c}0.745 \\
{[0.413]}\end{array}$ & $\begin{array}{c}0.882 \\
{[0.332]}\end{array}$ & 0.216 & 72 \\
\hline District includes non-targeted neighborhoods & $\begin{array}{c}0.400 \\
{[0.494]}\end{array}$ & $\begin{array}{c}0.176 \\
{[0.393]}\end{array}$ & 0.093 & 72 \\
\hline \multicolumn{5}{|l|}{ B. Retailer characteristics } \\
\hline Log-price index - pre-treatment (weighted) & $\begin{array}{l}-0.336 \\
{[0.084]}\end{array}$ & $\begin{array}{c}-0.324 \\
{[0.074]}\end{array}$ & 0.235 & 400 \\
\hline Percentage male & $\begin{array}{c}0.837 \\
{[0.370]}\end{array}$ & $\begin{array}{c}0.864 \\
{[0.345]}\end{array}$ & 0.543 & 401 \\
\hline 1 (if the surveyed person is the retailer's owner) & $\begin{array}{c}0.642 \\
{[0.480]}\end{array}$ & $\begin{array}{c}0.636 \\
{[0.484]}\end{array}$ & 0.897 & 401 \\
\hline 1 (if has more than a completed primary education) & $\begin{array}{c}0.623 \\
{[0.485]}\end{array}$ & $\begin{array}{c}0.659 \\
{[0.477]}\end{array}$ & 0.496 & 401 \\
\hline Log (total employees) & $\begin{array}{c}1.486 \\
{[0.461]}\end{array}$ & $\begin{array}{c}1.529 \\
{[0.513]}\end{array}$ & 0.397 & 401 \\
\hline Log (sales) & $\begin{array}{c}9.083 \\
{[0.822]}\end{array}$ & $\begin{array}{c}9.164 \\
{[0.865]}\end{array}$ & 0.429 & 388 \\
\hline Share of retailers in targeted neighborhood & $\begin{array}{c}0.601 \\
{[0.491]}\end{array}$ & $\begin{array}{c}0.784 \\
{[0.414]}\end{array}$ & 0.117 & 401 \\
\hline
\end{tabular}

Note: Columns [1] and [2] report the mean and standard deviation (in square brackets) of each variable at both the district and retailer level for compliers and non-compliers. Column [3] reports the p-value of a t-test of the difference between the two samples (using clustered standard errors at the district level). Column [4] shows the number of observations used.

*** Significant at the 1 percent level.

** Significant at the 5 percent level.

* Significant at the 10 percent level.

Source: Author's calculations 
APPENDIX TABLE A7. IMPACT ON INDIVIDUAL PRODUCT PRICES (ROBUSTNESS)

\begin{tabular}{|c|c|c|c|c|c|c|c|}
\hline \multirow[b]{2}{*}{ Outcome Log(Product Price) } & \multirow{2}{*}{$\begin{array}{c}\text { Weighting } \\
{[1]} \\
\end{array}$} & \multicolumn{2}{|c|}{ All } & \multicolumn{2}{|c|}{ Targeted } & \multicolumn{2}{|c|}{ Incumbents } \\
\hline & & $\begin{array}{r}\text { ITT } \\
{[2]} \\
\end{array}$ & $\begin{array}{l}\text { IV } \\
{[3]}\end{array}$ & $\begin{array}{r}\text { ITT } \\
{[4]} \\
\end{array}$ & $\begin{array}{l}\text { IV } \\
{[5]} \\
\end{array}$ & $\begin{array}{r}\text { ITT } \\
{[6]} \\
\end{array}$ & IV \\
\hline Rice (lb.) & 0.293 & $\begin{array}{c}-0.008 \\
{[0.013]}\end{array}$ & $\begin{array}{c}-0.015 \\
{[0.023]}\end{array}$ & $\begin{array}{c}-0.010 \\
{[0.017]}\end{array}$ & $\begin{array}{c}-0.022 \\
{[0.033]}\end{array}$ & $\begin{array}{c}-0.009 \\
{[0.018]}\end{array}$ & $\begin{array}{c}-0.022 \\
{[0.037]}\end{array}$ \\
\hline Cooking oil (lb.) & 0.094 & $\begin{array}{c}-0.030^{* *} * \\
{[0.015]}\end{array}$ & $\begin{array}{c}-0.057 \\
{[0.038]}\end{array}$ & $\begin{array}{c}-0.050 * * * \\
{[0.015]}\end{array}$ & $\begin{array}{c}-0.110 * * \\
{[0.046]}\end{array}$ & $\begin{array}{c}-0.052 * * * \\
{[0.016]}\end{array}$ & $\begin{array}{r}-0.129 * * \\
{[0.059]}\end{array}$ \\
\hline Sugar (lb.) & 0.052 & $\begin{array}{c}-0.001 \\
{[0.011]}\end{array}$ & $\begin{array}{c}-0.002 \\
{[0.020]}\end{array}$ & $\begin{array}{c}-0.003 \\
{[0.009]}\end{array}$ & $\begin{array}{c}-0.007 \\
{[0.020]}\end{array}$ & $\begin{array}{c}-0.008 \\
{[0.010]}\end{array}$ & $\begin{array}{c}-0.019 \\
{[0.023]}\end{array}$ \\
\hline Pasta (lb.) & 0.017 & $\begin{array}{c}-0.027^{* *} * \\
{[0.013]}\end{array}$ & $\begin{array}{c}-0.051 * * \\
{[0.024]}\end{array}$ & $\begin{array}{c}-0.048 * * * \\
{[0.015]}\end{array}$ & $\begin{array}{c}-0.102 * * \\
{[0.047]}\end{array}$ & $\begin{array}{c}-0.048 * * \\
{[0.016]}\end{array}$ & $\begin{array}{c}-0.113^{*} \\
{[0.058]}\end{array}$ \\
\hline Eggs (unit) & 0.050 & $\begin{array}{c}-0.022 \\
{[0.026]}\end{array}$ & $\begin{array}{c}-0.042 \\
{[0.044]}\end{array}$ & $\begin{array}{c}-0.025 \\
{[0.023]}\end{array}$ & $\begin{array}{c}-0.055 \\
{[0.046]}\end{array}$ & $\begin{array}{l}-0.025 \\
{[0.022]}\end{array}$ & $\begin{array}{l}-0.059 \\
{[0.052]}\end{array}$ \\
\hline Powdered milk (125 gr.) & 0.062 & $\begin{array}{c}0.032 \\
{[0.023]}\end{array}$ & $\begin{array}{c}0.060 \\
{[0.042]}\end{array}$ & $\begin{array}{c}0.019 \\
{[0.025]}\end{array}$ & $\begin{array}{c}0.040 \\
{[0.054]}\end{array}$ & $\begin{array}{c}0.006 \\
{[0.022]}\end{array}$ & $\begin{array}{c}0.015 \\
{[0.053]}\end{array}$ \\
\hline Chocolate (unit) & 0.015 & $\begin{array}{c}0.002 \\
{[0.011]}\end{array}$ & $\begin{array}{c}0.004 \\
{[0.021]}\end{array}$ & $\begin{array}{c}-0.008 \\
{[0.014]}\end{array}$ & $\begin{array}{c}-0.017 \\
{[0.028]}\end{array}$ & $\begin{array}{c}-0.009 \\
{[0.014]}\end{array}$ & $\begin{array}{c}-0.022 \\
{[0.030]}\end{array}$ \\
\hline Sardines (unit) & 0.014 & $\begin{array}{c}0.028 \\
{[0.032]}\end{array}$ & $\begin{array}{c}0.053 \\
{[0.062]}\end{array}$ & $\begin{array}{c}0.015 \\
{[0.044]}\end{array}$ & $\begin{array}{c}0.032 \\
{[0.097]}\end{array}$ & $\begin{array}{c}0.017 \\
{[0.042]}\end{array}$ & $\begin{array}{c}0.040 \\
{[0.100]}\end{array}$ \\
\hline Green beans (lb.) & 0.063 & $\begin{array}{l}-0.005 \\
{[0.006]}\end{array}$ & $\begin{array}{c}-0.009 \\
{[0.011]}\end{array}$ & $\begin{array}{c}-0.005 \\
{[0.008]}\end{array}$ & $\begin{array}{c}-0.011 \\
{[0.017]}\end{array}$ & $\begin{array}{c}-0.003 \\
{[0.008]}\end{array}$ & $\begin{array}{c}-0.007 \\
{[0.020]}\end{array}$ \\
\hline Onions (lb.) & 0.020 & $\begin{array}{c}-0.013 \\
{[0.022]}\end{array}$ & $\begin{array}{c}-0.024 \\
{[0.044]}\end{array}$ & $\begin{array}{c}-0.047 * * \\
{[0.022]}\end{array}$ & $\begin{array}{c}-0.104 * \\
{[0.062]}\end{array}$ & $\begin{array}{c}-0.038 * \\
{[0.022]}\end{array}$ & $\begin{array}{c}-0.092 \\
{[0.066]}\end{array}$ \\
\hline Salami (lb.) & 0.048 & $\begin{array}{c}-0.051 * \\
{[0.028]}\end{array}$ & $\begin{array}{c}-0.096^{*} \\
{[0.054]}\end{array}$ & $\begin{array}{c}-0.060 \\
{[0.039]}\end{array}$ & $\begin{array}{c}-0.132 \\
{[0.091]}\end{array}$ & $\begin{array}{l}-0.046 \\
{[0.040]}\end{array}$ & $\begin{array}{c}-0.111 \\
{[0.099]}\end{array}$ \\
\hline Chicken (lb.) & 0.170 & $\begin{array}{l}-0.014 \\
{[0.009]}\end{array}$ & $\begin{array}{c}-0.023 \\
{[0.017]}\end{array}$ & $\begin{array}{c}-0.008 \\
{[0.014]}\end{array}$ & $\begin{array}{l}-0.016 \\
{[0.025]}\end{array}$ & $\begin{array}{l}-0.008 \\
{[0.014]}\end{array}$ & $\begin{array}{l}-0.016 \\
{[0.028]}\end{array}$ \\
\hline Cod (lb.) & 0.018 & $\begin{array}{c}-0.010 \\
{[0.009]}\end{array}$ & $\begin{array}{c}-0.019 \\
{[0.016]}\end{array}$ & $\begin{array}{c}-0.020^{*} * \\
{[0.010]}\end{array}$ & $\begin{array}{c}-0.045 \\
{[0.031]}\end{array}$ & $\begin{array}{c}-0.023^{* *} \\
{[0.010]}\end{array}$ & $\begin{array}{c}-0.057 \\
{[0.039]}\end{array}$ \\
\hline Flour (lb.) & 0.010 & $\begin{array}{c}-0.038^{* *} \\
{[0.015]}\end{array}$ & $\begin{array}{c}-0.066^{* *} \\
{[0.031]}\end{array}$ & $\begin{array}{c}-0.042 * * \\
{[0.020]}\end{array}$ & $\begin{array}{c}-0.086^{*} \\
{[0.051]}\end{array}$ & $\begin{array}{c}-0.040 * \\
{[0.021]}\end{array}$ & $\begin{array}{c}-0.092 \\
{[0.060]}\end{array}$ \\
\hline Bread (unit) & 0.074 & $\begin{array}{l}0.088^{*} \\
{[0.052]}\end{array}$ & $\begin{array}{c}0.151 \\
{[0.107]}\end{array}$ & $\begin{array}{c}0.021 \\
{[0.057]}\end{array}$ & $\begin{array}{c}0.039 \\
{[0.107]}\end{array}$ & $\begin{array}{c}0.035 \\
{[0.057]}\end{array}$ & $\begin{array}{c}0.073 \\
{[0.121]}\end{array}$ \\
\hline
\end{tabular}

Note: Each entry shows an estimate of the impact of an increase in competition on the price of different products. Column [1] shows the weighting of each product in the final retailer price. Columns [2]-[3] use all the retailers; columns [4]-[5] use retailers in targeted neighborhoods; and columns [6]-[7] use incumbent retailers in targeted neigborhoods. All columns report the estimations while controlling for the baseline $\log ($ price $)$.

*** Significant at the 1 percent level.

** Significant at the 5 percent level.

* Significant at the 10 percent level. 